\title{
Processo de enfermagem: vantagens e desvantagens para a prática clínica do enfermeiro
}

RESUMO | A pesquisa tem como objetivo identificar as vantagens e as desvantagens relacionadas à realização do processo de enfermagem (PE) pelos enfermeiros que atuam nas unidades de internação em um hospital público de ensino. Trata-se de um estudo descritivo com análise quantitativa dos dados. A coleta de dados foi realizada no período de outubro de 2016 a março de 2017 por meio de entrevista individual com enfermeiros que aceitaram fazer parte do estudo, totalizando 29 sujeitos. Foram apontados como eventos vantajosos decorrentes da realização do PE: cuidado humanizado, individualizado e holístico; direcionamento da prática e tomada de decisão; cuidado qualificado; maior qualidade dos registros de enfermagem; valorização profissional; melhoria na condição de saúde dos clientes; autonomia profissional; maior interação da equipe de enfermagem; satisfação do usuário e maior controle de custos. Os aspectos negativos decorrentes da realização do PE foram: necessidade de acompanhamento sequencial do paciente, necessidade de maior número de enfermeiros assistenciais e maior disponibilidade de tempo para avaliar, planejar, prescrever, executar e registrar as respostas do paciente no prontuário, no entanto, tais itens consistem em "dificuldades" para a realização do PE e não como "desvantagens" da realização do mesmo. Foi possível verificar que a realização do PE traz inúmeras vantagens para o paciente, profissional e instituição de saúde e nenhuma desvantagem aos envolvidos nesse processo.

Palavras-chaves: Planejamento de Assistência ao Paciente, Avaliação de Enfermagem, Processos de Enfermagem.

ABSTRACT | The research aims to identify the advantages and disadvantages related to the performance of the nursing process (PE) by nurses working in inpatient units in a public teaching hospital. This is a descriptive study with quantitative data analysis. Data collection was performed from October 2016 to March 2017 through individual interviews with nurses who agreed to be part of the study, totaling 29 subjects. Advantageous events resulting from the performance of the PE were: humanized, individualized and holistic care; practice direction and decision making; qualified care; higher quality of nursing records; professional appreciation; improvement in clients' health condition; professional autonomy; greater interaction of the nursing staff; user satisfaction and greater cost control. The negative aspects resulting from the performance of PE were: need for sequential patient follow-up, need for more nurses and more time available to evaluate, plan, prescribe, execute and record patient responses in the medical record; These items consist of "difficulties" for performing PE and not as "disadvantages" of performing PE. It was possible to verify that the performance of PE brings numerous advantages for the patient, professional and health institution and no disadvantage to those involved in this process. Keywords: Patient Care Planning, Nursing Assessment, Nursing Processes.

RESUMEN | La investigación tiene como objetivo identificar las ventajas y desventajas relacionadas con el desempeño del proceso de enfermería (EP) por parte de las enfermeras que trabajan en unidades de hospitalización en un hospital público de enseñanza. Este es un estudio descriptivo con análisis de datos cuantitativos. La recopilación de datos se realizó entre octubre de 2016 y marzo de 2017 a través de entrevistas individuales con enfermeras que aceptaron ser parte del estudio, totalizando 29 sujetos. Los eventos ventajosos resultantes del desempeño de la EP fueron: atención humanizada, individualizada y holística; dirección práctica y toma de decisiones; cuidado calificado; mayor calidad de los registros de enfermería; apreciación profesional; mejora en el estado de salud de los clientes; autonomía profesional; mayor interacción del personal de enfermería; satisfacción del usuario y mayor control de costos. Los aspectos negativos resultantes del desempeño de la EP fueron: necesidad de seguimiento secuencial del paciente, necesidad de más enfermeras y más tiempo disponible para evaluar, planificar, prescribir, ejecutar y registrar las respuestas de los pacientes en la historia clínica; Estos elementos consisten en "dificultades" para realizar PE y no como "desventajas" de realizar PE. Fue posible verificar que el desempeño de la EP trae numerosas ventajas para el paciente, el profesional y la institución de salud y no una desventaja para los involucrados en este proceso.

Descriptores: Planificación de la Atención del Paciente, Avaluación de Enfermería, Procesos de Enfermería.

\section{Djulia Camila Berwanger}

Enfermeira. Graduada pela Universidade Estadual do Oeste do Paraná, campus Cascavel/PR.

Fabiana Gonçalves de Oliveira Azevedo Matos

Enfermeira. Doutora. Docente da Universidade Estadual do Oeste do Paraná, campus Cascavel/PR

\section{João Lucas Campos de Oliveira}

Enfermeiro. Doutor. Docente da Universidade Federal de Mato Grosso. Cuiabá/MT.

\section{Débora Cristina Ignácio Alves}

Enfermeira. Doutora. Docente da Universidade Estadual do Oeste do Paraná, campus Cascavel/PR.

\section{Lili Marlene Hofstatter}

Enfermeira. Mestre. Docente da Universidade Estadual do Oeste do Paraná, campus Cascavel/PR.

\section{Nelsi Salete Tonini}

Enfermeira. Doutora. Docente da Universidade Estadual do Oeste do Paraná, campus Cascavel/PR.

\section{Akie Fujii Neta \\ Enfermeira. Graduada pela Universidade Estadual do Oeste do Paraná, campus Cascavel/PR.}

Recebido em: 08/08/2019

Aprovado em: 28/08/2019 
INTRODUÇÃO

A estruturação da enfermagem como profissão sofre influências do contexto econômico, político, social e cultural que por sua vez, determinam suas práticas e direcionam seu processo de trabalho atuação ${ }^{1}$. A profissão de enfermagem surgiu do desenvolvimento e da evolução das práticas de saúde no decorrer do tempo, desenvolvendo suas atividades de forma repetitiva, executava ordens e realizava procedimentos padronizados com base em normas e rotinas, sem refletir sobre sua atuação ${ }^{2}$.

As primeiras tentativas de sistematizar o cuidado de enfermagem por meio da introdução do Processo de Enfermagem (PE) nas instituições de saúde e no ensino de graduação brasileiro ocorreram no final da década de 1970 e início de 1980 por Wanda Horta e trouxeram mudanças positivas ao referido cenário, pois possibilitou ao enfermeiro a adoção de um método de trabalho sistematizado baseado em conhecimento científico ${ }^{2}$. No entanto foi somente em 1986, com a Lei $n^{\circ} 7.498 / 86$ que regulamenta o Exercício Profissional da Enfermagem, que o PE passou a ser visto como uma atividade privativa do enfermeiro ${ }^{3}$.

A realização do $P E$ tornou-se obrigatória em todas as instituições de saúde no Brasil após o Conselho Federal de Enfermagem (COFEN) estabelecer a Resolução $n^{\circ}$ 272/2002. Em 2009, o mesmo conseIho elaborou a Resolução n 358/2009, tornando obrigatória a estruturação da Sistematização da Assistência de Enfermagem (SAE) e a realização do PE para todos os ambientes, públicos ou privados, em que ocorre o cuidado profissional de enfermagem ${ }^{4}$.

No entanto, mesmo sendo uma exigência legal, o cotidiano de trabalho dos enfermeiros tem demonstrado que a nível nacional o PE ainda não se encontra totalmente implantado nos serviços de saúde ${ }^{5}$.

O PE é uma ferramenta valiosa para a otimização do desempenho institucional e necessária para a efetividade dos princípios e valores das políticas de saúde. Além de organizar e sistematizar o trabalho da equipe de enfermagem, esse método de trabalho contribui para a criação de um plano de eficácia de custos, auxilia na comunicação de enfermagem, diminui a margem de erros cometidos pelos profissionais, e acima de tudo, elabora um plano de cuidados individualizado ao sujeito assistido e não apenas à doença, direcionando as intervenções de enfermagem para a promoção, prevenção, recuperação e reabilitação da saúde ${ }^{6}$.

Diante do exposto, a pesquisa tem como objetivo identificar as vantagens e as desvantagens relacionadas à realização do PE pelos enfermeiros que atuam nas unidades de internação em um hospital público de ensino.

\section{MÉTODO}

Trata-se de um estudo descritivo, transversal com análise quantitativa dos dados, desenvolvido nas unidades de internação de um hospital público de ensino localizado no interior do Paraná. Trata-se de um hospital de médio porte, com 195 leitos, que atende as seguintes especialidades: Clínica Médica, Clínica Cirúrgica, Ginecologia e Obstetrícia, Pediatria, Neurologia e Ortopedia, Cardiovascular e Psiquiatria. Possui ainda atendimento complementar em: UTI Neonatal, UTI Geral, UTI Pediátrica, Unidade de Cuidados Intermediários e Pronto Socorro, sendo o único hospital público das Regiões Oeste e Sudoeste do Paraná com $100 \%$ de seus leitos destinados à pacientes do Sistema Único de Saúde (SUS). É considerado hospital de referência dessa região, com uma média mensal de 833 internações.

Os dados foram coletados por meio de entrevista individual, realizada nos meses de outubro de 2016 e março de 2017. Os critérios de inclusão no estudo foram: ser enfermeiro e não estar afastado das atividades laborais no momento da coleta de dados. Com base na planilha de enfermeiros fornecida pela direção de enfermagem do hospital pesquisado, a população do estudo seria composta por 66 enfermeiros, no entanto, apenas 29 enfermeiros concordaram em participar da pesquisa por meio da assinatura do Termo de Consentimento Livre e Esclarecido (TCLE).

Os dados das entrevistas foram analisados e transcritos para uma planilha eletrônica do programa Microsoft Office Excel 2010, cujos resultados foram apresentados por meio de frequência absoluta e relativa, dispostos em tabelas.

Este estudo é parte do projeto de pesquisa intitulado "Sistematização da assistência de enfermagem: implantação na prática clínica de um Hospital Escola", aprovado pelo Comitê de Ética em Pesquisa da Universidade Estadual do Oeste do Paraná (UNIOESTE), CAAE: 19704613.1.0000.0107.

\section{RESULTADOS}

Com relação à caracterização da amostra, a maioria dos entrevistados tinha idade entre 30 a 39 anos ( $n=20 ; 68,9 \%$ ), era do sexo feminino $(n=23 ; 79,3 \%)$, tinha recebido conteúdo sobre $\mathrm{PE}$ na graduação ( $n=27 ; 93,2 \%)$, tinha moderado conhecimento sobre PE ( $n=18 ; 62,1 \%)$ e não realizava o PE no seu cotidiano de trabalho $(\mathrm{n}=16 ; 55,2 \%)$ (Tabela 1$)$.

\begin{tabular}{|c|c|c|}
\hline Descrição & $\mathbf{n}$ & $\%$ \\
\hline \multicolumn{3}{|l|}{ Idade } \\
\hline 20-29 anos & 03 & $10,3 \%$ \\
\hline 30-39 anos & 20 & $68,9 \%$ \\
\hline 40-49 anos & 05 & $17,3 \%$ \\
\hline
\end{tabular}




\begin{tabular}{|c|c|c|}
\hline 50-59 anos & 01 & $3,5 \%$ \\
\hline \multicolumn{3}{|l|}{ Gênero } \\
\hline Masculino & 06 & $20,7 \%$ \\
\hline Feminino & 23 & $79,3 \%$ \\
\hline \multicolumn{3}{|c|}{ Conteúdo sobre PE na graduação } \\
\hline $\operatorname{Sim}$ & 27 & $93,2 \%$ \\
\hline Não & 02 & $6,8 \%$ \\
\hline \multicolumn{3}{|c|}{ Conhecimento sobre PE } \\
\hline Pequeno & 08 & $27,6 \%$ \\
\hline Moderado & 18 & $62,1 \%$ \\
\hline Grande & 03 & $10,3 \%$ \\
\hline \multicolumn{3}{|c|}{ Realização do PE no trabalho } \\
\hline $\operatorname{Sim}$ & 13 & $44,8 \%$ \\
\hline Não & 16 & $55,2 \%$ \\
\hline
\end{tabular}

A Tabela 2 apresenta a frequência das vantagens pré-selecionadas no instrumento de coleta de dados elaborado à partir da literatura, resultantes da realização do PE na prática clínica do enfermeiro.
Dos 29 (100\%) enfermeiros que participaram do estudo, apenas três (10,3\%) apontaram que a realização do PE na prática clínica causa algum evento desvantajoso, evidenciado na Tabela 3.

Tabela 2 - Distribuição das vantagens identificadas na realização do PE pelos enfermeiros entrevistados. Cascavel, 2017.

Vantagens da realização do PE

Cuidado humanizado, individualizado e holístico

Direcionamento da prática e tomada de decisão

Cuidado qualificado

Maior qualidade dos registros de enfermagem

Valorização profissional

Melhoria na condição de saúde dos clientes

Autonomia profissional

Maior interação da equipe de enfermagem

Satisfação do usuário

Fonte: dados da pesquisa

Tabela 3 - Distribuição das desvantagens identificadas na realização do PE pelos enfermeiros entrevistados. Cascavel, 2017.

\section{Desvantagens da realização do PE}

Necessidade de acompanhamento sequencial do paciente

Necessidade de maior quantitativo de enfermeiros

Necessidade de mais tempo para avaliação, planejamento, prescrição, execução e registro

$\mathrm{N} \%$

$28 \quad 96,5$

$27 \quad 93,2$

$26 \quad 89,7$

$25 \quad 86,2$

$24 \quad 82,7$

$24 \quad 82,7$

$23 \quad 79,3$

$23 \quad 79,3$

$20 \quad 68,9$

Fonte: dados da pesquisa.
Discussão

1) Caracterização da amostra:

Com relação à caracterização da amostra, a maioria dos entrevistados tinha idade entre 30 a 39 anos $(n=20$; $68,9 \%)$, era do sexo feminino $(n=23$; $79,3 \%$ ), tinha recebido conteúdo sobre PE na graduação $(n=27 ; 93,2 \%)$, tinha moderado conhecimento sobre PE $(\mathrm{n}=18 ; 62,1 \%)$ e não realizava o $\mathrm{PE}$ no seu cotidiano de trabalho $(n=16 ; 55,2 \%)$ (Tabela 1). Estudo realizado com enfermeiros das cinco regiões brasileiras identificou que a maioria $(49,7 \%)$ também tinha menos de 40 anos de idade, com predominância do sexo feminino na categoria de enfermeiros ${ }^{7}$. Com relação a ter recebido ou não conteúdo sobre PE na graduação, como sendo uma forma de otimizar a realização do PE na prática clínica, é importante destacar que as dificuldades para a realização do PE já se iniciam-se ainda durante o ensino na graduação ${ }^{8}$. Com relação à realização do PE no cotidiano de trabalho, o presente estudo identificou que apenas $44,8 \%$ dos enfermeiros realizam o PE na sua rotina de trabalho. O despreparo e a falta de interesse dos profissionais de enfermagem tornam-se obstáculos à efetiva execução do PE, fazendo com que os enfermeiros se limitem em cumprir as normas estabelecidas pela instituição ${ }^{9}$.

2) Vantagens da realização do PE na prática clínica:

Quase a totalidade dos enfermeiros entrevistados $(n=28 ; 96,5 \%)$ responderam que o "cuidado humanizado, individualizado e holístico" é uma vantagem decorrente da realização do PE na prática clínica. Por meio do PE, o enfermeiro consegue organizar seu trabalho com base em um referencial teórico e em um método científico que prioriza a individualidade do cuidado ${ }^{8}$. Quando o PE é realizado de forma correta, torna-se um processo articulador, possibilitando uma melhor avaliação do paciente, enfocando o cuidado ao indivíduo inserido em um contexto de vida, onde o cuidado é integral, contínuo e voltado para o sujei- 
to e não para a doença ${ }^{9}$

O "direcionamento da prática de enfermagem e o favorecimento da tomada de decisão" foi apontado por 93,2\% $(n=27)$ dos enfermeiros entrevistados. Por meio da realização do PE, o enfermeiro desenvolve o julgamento clínico necessário para a identificação dos diagnósticos de enfermagem que favorece a tomada de decisão, a implementação e a avaliação do cuidado prestado ${ }^{5}$. O PE fornece ainda, sustentação teórica aplicada a prática, sistematizando a assistência e direcionando as ações da equipe de enfermagem para a correta execução dos cuidados prestados ${ }^{5}$.

O "cuidado qualificado" foi apontado por $89,7 \%(n=26)$ dos enfermeiros entrevistados. A execução do PE proporciona melhorias na qualidade da assistência prestada por meio do desenvolvimento de relações humanizadas entre enfermeiros e usuários e por meio da valorização de princípios éticos ${ }^{5}$.

Por sua vez, o PE representa um importante instrumento técnico-científico capaz de promover a qualidade e a continuidade da assistência de enfermagem $^{6,10}$. Da mesma forma, a realização do PE promove uma assistência de enfermagem mais segura, dinâmica, competente e organizada ${ }^{6}$.

A "otimização dos registros e recuperação de dados" foi apontada por $86,2 \% \quad(n=25)$ dos enfermeiros entrevistados. A realização do PE favorece a documentação da assistência prestada visto que beneficia o preenchimento dos registros e o armazenamento dos dados relacionados ao cliente ${ }^{11}$.

Ao registrar as informações e ações realizadas com os pacientes assistidos, em seus respectivos prontuários, os enfermeiros informam qual foi a contribuição da enfermagem na recuperação dos mesmos. Tais dados poderão ser analisados posteriormente para fins de pesquisas, auditoria, avaliação de qualidade em serviço ${ }^{12}$.

A "valorização profissional" foi apontada por $82,7 \% \quad(n=24)$ dos enfer- meiros entrevistados. A realização do $\mathrm{PE}$ promove maior valorização profissional, não só pela distinção e reconhecimento do trabalho da enfermagem por outros profissionais, mas pela satisfação de ver seu próprio trabalho proporcionar bons resultados aos pacientes ${ }^{8}$.

A "melhoria na condição de saúde dos clientes" foi apontada por 82,7\% $(n=24)$ dos enfermeiros entrevistados. A realização do PE favorece a identificação de situações de saúde/doença por meio de métodos e estratégias de trabalho científico, contribuindo para a promoção, prevenção e recuperação da saúde do indivíduo, da família e/ou da comunidade, promovendo melhorias na condição de saúde dos envolvidos nesse processo $^{2}$.

Quando a assistência é planejada, é possível diagnosticar as necessidades do cliente favorecendo uma prescrição adequada dos cuidados de enfermagem. Espera-se que, com a correta realização do $\mathrm{PE}$, seja possível que o indivíduo assistido tenha melhorias na autoestima, identificação de novos valores, espiritualidade, efetivação da busca pela saúde, adaptação psicossocial e, consequentemente, mudanças no estilo de vida ${ }^{13}$.

A "autonomia profissional" foi apontada por $79,3 \%(n=23)$ dos enfermeiros entrevistados. A realização do PE proporciona ao enfermeiro maior autonomia profissional, tornando possível a aplicação de seus conhecimentos na prática clínica, conquistando o reconhecimento profissional pela qualidade do cuidado prestado ao indivíduo, família ou comunidade, no entanto, é importante que o enfermeiro tenha fundamentação teórica e atitudes assertivas para manter a autonomia adquirida $^{14}$.

A "maior interação da equipe de enfermagem" foi apontada por 79,3\% $(n=23)$ dos enfermeiros entrevistados. A realização do PE favorece as relações interpessoais promovendo uma maior interação do enfermeiro com a clientela as- sistida e com a equipe multidisciplinar. Um dos desafios para a implementação do PE é justamente a sensibilização da equipe de enfermagem sobre a importância do mesmo, sendo importante que o enfermeiro promova ações educativas para que toda a equipe de enfermagem reconheça a importância dessa metodologia e desenvolva meios para viabilizar a execução do PE na prática clínica de enfermagem ${ }^{15}$.

A "satisfação do usuário" foi apontada por $68,9 \%(n=20)$ dos enfermeiros entrevistados. A avaliação da satisfação do cliente tem sido adotada por algumas instituições de saúde que utilizam o PE como estratégia para obter informações relacionadas à qualidade da assistência prestada $^{16}$.

Estudo revela que os pacientes demonstraram satisfação frente ao cuidado recebido, pois eram assistidos em sua singularidade, sob uma visão holística, que permitia um cuidado de qualidade e integral proporcionado por meio da realização do $\mathrm{PE}^{17}$.

3) Desvantagens da realização do PE na prática clínica

As desvantagens levantadas pelos enfermeiros entrevistados eram na verdade, itens necessários para a realização do PE (Tabela 3) podendo ser entendidas como "dificuldades" para a realização do PE e não como "desvantagens" da realização do mesmo.

Santos e Santos (2012) corroboram com a presente pesquisa, enfatizando que de forma geral, não há desvantagens decorrentes da realização do PE na prática clínica do enfermeiro ${ }^{18}$.

Segundo os dados coletados e os dados repassados pela direção de enfermagem do hospital, no período de estudo existia uma sobrecarga de trabalho para a enfermagem em todos os setores do hospital por falta de funcionários (auxiliares, técnicos e enfermeiros). Além do quantitativo reduzido, ainda existia servidores afastados por acidente de trabalho, com restrição de atividades, em férias ou em licenças. 
Constituíram limitações do estudo o fato da coleta de dados ter sido realizada em uma única instituição de saúde, que ainda não tinha o PE efetivamente implantado na rotina de trabalho dos enfermeiros, fazendo com que as experiências vivenciadas pelos enfermeiros entrevistados fossem decorrentes de um mesmo cenário.

\section{CONCLUSÃO}

O PE consiste em um método científico de trabalho que relaciona as teorias de enfermagem com a prática assistencial. A realização do PE permite que o enfermeiro tome decisões baseadas em fatos, direcionando as suas ações e otimizando o trabalho da enfermagem.

Assim como evidenciado pelo presente estudo, a literatura científica mostra que a realização do PE traz inúmeros benefícios para a enfermagem, para a equipe multiprofissional, para a instituição e principalmente para o paciente e sua família. No entanto, o simples fato de realizá-lo não garante que o cuidado prestado seja individualizado e qualificado. É necessário que haja comprometimento de toda equipe de enfermagem, que o enfermeiro tenha profundo conhecimento teórico e prático de todas as etapas que constituem o PE e que a instituição onde ocorra o cuidado viabilize a realização do mesmo, garantindo um adequado número de enfermeiros e oferecendo condições adequadas de trabalho. É preciso refletir e discutir conjuntamente sobre os obstáculos que a grande maioria dos enfermeiros encontra frente a operacionalização do PE para que seja possível a sua efetiva implantação na prática clínica do enfermeiro.

\section{Referências}

1. Silva MV, Figueiredo MLF. Desafios históricos da enfermagem à luz do pensamento bioético. Rev. Bras. Enferm. [Internet]. 2010 [acesso em 2017 mai 28]; 63(5): 841-3. Disponível em: http://www.scielo. $\mathrm{br} /$ readcube/epdf.php?doi=10.1590/S0034-71672010000500024\&pi$d=$ S003471672010000500024\&pdf_path=reben/v63n5/24.pdf

2. Marques SM, Brito KCG, Fernandes CM, Vieira AG. Sistematização da assistência de enfermagem na UTI: perspectivas dos enfermeiros da cidade de Governador Valadares. REME [Internet]. 2008 [acesso em 2017 mai 28]; 12(4): 469-476. Disponível em: http://www.reme.org.br/artigo/detalhes/290 3. Mangueira SO, Lima JTS, Costa SLA, Nóbrega MML, Lopes MVO. Implantação da sistematização da assistência de enfermagem: opinião de uma equipe de enfermagem hospitalar. Enfermagem em Foco [Internet]. 2012 [acesso em 2017 jun 07] 3(3): 135-8. Disponível em: <http://revista.portalcofen.gov.br/ index.php/enfermagem/article/viewFile/298/160>.

4. Conselho Federal de Enfermagem. Resolução COFEN n³58/2009 de 15 de outubro de 2009. Dispõe sobre a sistematização da assistência de enfermagem - SAE nas instituições de saúde brasileiras. Rio de Janeiro: Conselho Federal de Enfermagem [Internet]. 2009 [acesso em 2017 abr 15]. Disponível em: http://revista.cofen.gov.br/index.php/enfermagem/article/viewFile/298/160

5. Medeiros AL, Santos SR, Cabral RW. Sistematização da assistência de enfermagem na perspectiva dos enfermeiros: uma abordagem metodológica na teoria fundamentada. Revista Gaúcha de Enfermagem [Internet]. 2012 [acesso em 2017 jul 19]; 33(3):174-181. Disponível em: http://www.scielo.br/scielo. php?script=sci_arttext\&pid=S1983-14472012000300023

6. Amante LN, Rossetto, AP Schneider DG. Sistematização da assistência de enfermagem em unidade de terapia intensiva sustentada pela teoria de Wanda Horta. Revista da Escola de Enfermagem USP [Internet]. 2009 [acesso em 2017nov 09]; 43 (1):54-64. Disponível em: http://www.scielo.br/pdf/reeusp/ v43n1/07.pdf

7. Menzani G, Bianchi ERF. Stress dos enfermeiros de pronto socorro dos hospitais brasileiros. Revista Eletrônica de Enfermagem [Internet] 2009 [acesso em 2017 set 30] 11(2): 327-33. Disponível em: https://www.fen.ufg.br/fen revista/v11/n2/v11n2a13.htm

8. Luiz FF, Padoin SMM, Neves ET, Ribeiro AC, Tronco CS. A sistematização da assistência de enfermagem na perspectiva da equipe de um hospital de ensino. Rev. Eletr. Enf. [Internet]. 2010 [acesso em 2017 jun 14]; 12(4): 655-9. Disponivel em: http://www.fen.ufg.br/revista/v12/n4/v12n4a09.htm

9. Castilho NC, Ribeiro PC, Chirelli MQ. A implementação da sistematização da assistência de enfermagem no serviço de saúde hospitalar do Brasil. Texto Contexto Enfermagem [Internet]. 2009 [acesso em 2017 abr 15]; 18(2): 280-9. Disponivel em: http://www.scielo.br/pdf/tce/v18n2/11.pdf

10. Gabriel CS, Gabriel AB, Bernardes A, Rocha FLR, Miasso Al. Qualidade na assistência de enfermagem hospitalar: visão de alunos de graduação. Revista Gaúcha Enfermagem [Internet]. 2010 [acesso em 2017 abr 27]; 31(3): 529-35. Disponivel em: http://www.scielo.br/pdf/rgenf/v31n3/v31n3a17.pdf 11. Oliveira SM, Ribeiro RCHM, Ribeiro DF, Lima LCEQ, Pinto MH, Poletti NAA. Elaboração de um instrumento da assistência de enfermagem na unidade de hemodiálise. Acta Paulista de Enfermagem [Internet]. 2008 [acesso em 2017 abr 27]; 21(Número Especial):169-73. Disponível em: http://www.scielo.br/ pdf/ape/v21nspe/a06v21ns.pdf

12. Barra DCC, Sasso GTMD. Padrões de dados, terminologias e sistemas de classificação para o cuidado em saúde e enfermagem. Rev Bras Enfermagem [Internet]. 2011 [acesso em 2015 abr 15]; 64(6): 1141-9. Disponível em: http:/l www.scielo.br/pdf/reben/v64n6/v64n6a23.pdf

13. Teixeira GA, Carvalho JBL, Silva ALMA, Santos SB, Lopes TRG. Sistematização da assistência de enfermagem a pessoa em situação de rua. Rev Enferm UFPE on line [Internet]. 2015 [acesso em 2015 abr 15]; 9(3): 7169-74. Disponivel em: https://www.google.com/url?sa=t\&rct=j\&q=\&esrc=s\&sour$c e=$ web\&cd=2\&ved=2ahUKEwiE9_OJr5nkAhVvFbkGHVZrD9EQFjABegQIABAC\&url=https $\% 3 \mathrm{~A} \% 2 \mathrm{~F} \% 2 \mathrm{Fperiodicos}$.ufpe.br\%2Frevistas $\% 2$ Frevistaenfermagem $\% 2$ Farticle $\% 2$ Fdownload $\% 2 F 10448 \% 2 F 11263 \&$ usg=AOVVaw1 r8AE8-YRCmddf2Ujo3saj

14. Menezes SRT, Priel MR, Pereira LL. Autonomia e vulnerabilidade do enfermeiro na prática da Sistematização da Assistência de Enfermagem. Rev Esc. Enferm. USP [Internet]. 2011 [acesso em 2017 abr 16]; 45 (4): 953-8. Disponível em: http://www.scielo.br/pdf/reeusp/v45n4/v45n4a23.pdf

15. Hermida PMV. Desvelando a implementação da sistematização da assistência de enfermagem. Revista Brasileira de Enfermagem [Internet]. 2004 [acesso em 2017 abr 13]; 57(6): 733-7. Disponível em: http://dx.doi. org/10.1590/S0034-71672004000600021

16. Lyu H, Wick EC, Housman M, Freischlag JA, Makary MA. Patient satisfaction as a possible indicator of quality surgical care. JAMA Surg. [Internet]. 2013 [acesso em 2017 out 02]; 148(4):362-7. Disponível em: http://archsurg. jamanetwork.com/article. aspx?articleid $=1679648$

17. Penedo RM, Spiri WC. Significado da sistematização da assistência de enfermagem para enfermeiros gerentes. Acta Paul Enferm. [Internet]. 2014 [acesso em 2017 out 02]; 27(1):86-92. Disponível em: http://www.scielo.br/ scielo.php?pid=s0103-21002014000100016\&script=sci_arttext\&tlng=pt 18. Santos MIS, Santos WL. Uso da sistematização da assistência de enfermagem (SAE): uma ferramenta para realização da auditoria de qualidade. Revista de Divulgação Científica Sena Aires [Internet]. 2012 [acesso em 2017 out 02]; (2): 179-184. Disponível em: http://revistafacesa.senaaires.com.br/index. php/revisa/article/view/29 